\title{
The OSA-MIMO Technologies for Future Wireless Communications
}

\author{
J. Pawelec ${ }^{1, *}$, M. Suchański ${ }^{2}$, K. Kosmowski ${ }^{2}$, T. Szymczyk ${ }^{2}$ \\ ${ }^{1}$ Pulaski University of Technology \& Humanities, Radom, Poland \\ ${ }^{2}$ Military Communication Institute, Poland \\ *Corresponding author: j.pawelec@uthrad.pl
}

Copyright (C) 2013 Horizon Research Publishing All rights reserved.

\begin{abstract}
The article analyses the role of Multiple Input Multiple Output (MIMO) and Opportunity Spectrum Access (OSA) technologies in future Cognitive Radio (CR). The model of two networks operating in the same frequency band under Rayleigh fading conditions is studied in detail. The network of licensed users called the primary operates in SISO mode, while the secondary one - in MIMO mode. The results of the analysis are encouraging. For the future it is proposed that the networks should be equipped with a dense grid of spectrum sensing detectors and OSA-routers.
\end{abstract}

Keywords Spectrum Economy, Cognitive Radio, Opportunity Spectrum Access, MIMO Systems

\section{Introduction}

Since its appearance the OSA-MIMO idea has been struggling with a severe shortage of the EM spectrum and its wasteful exploitation by the licensed owners. According to the last reports they use the assigned spectrum no more than $10 \%$ of the time, on average [1], [2]. Due to the original Mitola's idea [1], the secondary users (SU) can transmit only, if the primary users (PU) are inactive. This is called the interweave strategy. There is also an underlay strategy, which allows SU to transmit even if the primary user is active. The only condition is that the secondary transmitter (ST) keeps its interfere power below the predefined threshold at each primary receiver (PR) [3]. This requires delimitation of the power allocation schemes, Fig.1

If we assume that primary users operate in a conventional SISO mode, while the secondary users in MIMO mode, the deficit of spectrum can be diametrically improved. This is because MIMO technology offers the new space channels. An architecture of $2 \times 2$ antennas creates 4 independent channels in the same frequency band. Then, the capacity theoretically increases four times.

The problem however arises in distinguishing of new channels at the receiver side. Some authors, e.g. Alamouti
[4], use for this purpose additional signals, say $\mathrm{s}_{1}{ }^{*}$ and $-\mathrm{s}_{2}$ parallel to $s_{1}$ and $s_{2}$, which help to identify the channel where the signal comes from. So, the effective transmission rate decreases. The resultant gain is, however, still positive and the capacity is proportional to the number of antennas at one side of a link (in case of MIMO2x2 - two times).

The power allocation for MIMO cognitive networks is studied in [4-6]. The OSA technology is analyzed in more detail in [7-8]. It means in practice a dense net of the spectrum sensing modules and the associated traffic routers should be introduced.

The remainder of this paper is as follows: an analysis of the simple OSA-MIMO scheme is described in chapter II, the rigorous model of advanced MIMO system - in chapter III, an analysis of Ad Hoc free access network - in chapter IV. Then, chapter $\mathrm{V}$ presents homogeneous MIMO network example and chapter VI - comparisons and conclusions.

\section{Simple SISO-MIMO Opportunity Scheme}

Let us consider two networks operating in the same frequency band and the same area, Fig.1. The primary network P operates in SISO mode, while the secondary $\mathrm{S}$ - in MIMO. The slow Rayleigh fading is accompanying. When the receiver $P_{R}$ experiences an excessive fading so that $\mathrm{SNR}_{\mathrm{P}}<1$, the network $\mathrm{S}$ starts to transmit data, assuming $\mathrm{SNR}_{\mathrm{S}}>1$. Occasionally, both may be blocked. The channels are duplex oriented and the transfer functions are gained via adaptation [5].

In order to simplify the model, the receiver noise in both networks is normalized to be equal to one, $\mathrm{N}_{0}=1$. If the useful signal $\mathrm{N}_{\mathrm{i}}$ exceeds the level $\mathrm{N}_{0}$, the network $\mathrm{P}$ starts to transmit data until $\mathrm{SNR}_{\mathrm{P}}<1$. Then, it is network $\mathrm{S}$ turn to transmit. It can be shown that a unit capacity $\mathrm{C}_{\mathrm{s}}$ is then as follows

$$
C_{s}=\frac{1}{M} \sum_{i=1}^{M} \log _{2}\left(1+N_{i} / N_{0}\right)
$$


where $\mathrm{M}$ - total number of signal samples (packets); $\mathrm{N}_{\mathrm{i}}-$ $i$-th packet power level $\left(\mathrm{SNR}_{\mathrm{S}}\right)$.

The obtained results are shown in Fig 2. As we can see the supplementary capacity $\mathrm{C}_{\mathrm{s}}$ of network $\mathrm{S}$ reaches quite large values. The highest $\sim 9 \mathrm{~b} / \mathrm{s} / \mathrm{Hz}$ obtained for the 4 -th order MIMO (1Tx-4Rx) is much greater than the capacity of an individual SISO channel without fading $(6.6 \mathrm{~b} / \mathrm{s} / \mathrm{Hz})$.

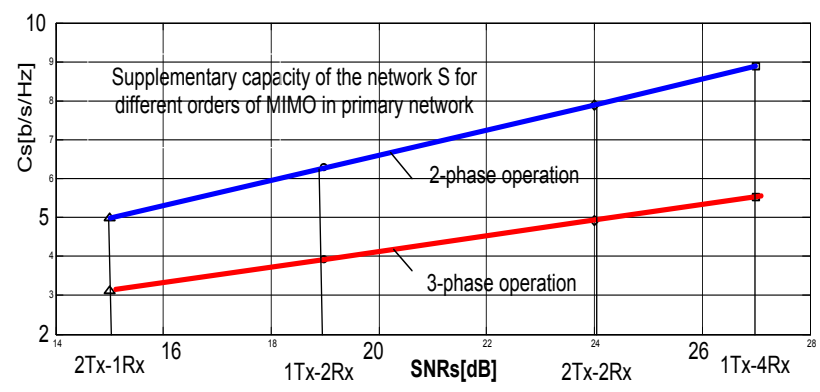

Figure 1. Capacity $\mathrm{C}_{\mathrm{s}}$ of the secondary network vs SNR under SISO/MIMO rivalry for two schemes of traffic organization

The lower curve (red) in Fig.2 corresponds to the following 3-state Rayleigh scenario: (1) network P transmits, (2) network S transmits, (3) both networks are blocked. The blue curve corresponds to the case of the state (3) when $\mathrm{MIMO}$ is used for $\mathrm{S}$ transmission via higher $\mathrm{SNR}_{\mathrm{S}}$. The more general approach of the problem is done in [9]

$$
\mathbf{y}_{s}=\mathbf{H} \mathbf{x}_{\mathbf{s}}+\sum_{i} \mathbf{T}_{\mathbf{i}} \mathbf{x}_{\mathrm{p}, \mathrm{i}}+\mathbf{n}_{\mathbf{0}}
$$

$\mathbf{y}_{\mathrm{s}}-$ total received signal vector at the secondary receiver $\left(\mathrm{S}_{\mathrm{R}}\right) ; \mathbf{x}_{\mathrm{s}}$ - useful signal vector of the secondary transmitter $\left(\mathrm{S}_{\mathrm{T}}\right), \mathbf{H}-\mathrm{MIMO}$ channel matrix from $\mathrm{S}_{\mathrm{T}}$ to $\mathrm{S}_{\mathrm{R}} ; \mathbf{T}_{\mathrm{i}}$ - channel matrix from the primary transmitter $\mathrm{P}_{\mathrm{T}}$ to the secondary receivers $\mathrm{S}_{\mathrm{R}} ; \mathbf{x}_{\mathrm{p}, \mathrm{I}}-$ signal vector at i-th transmitter $\mathrm{P}_{\mathrm{T}} ; \mathbf{n}_{0}-$ normalized Gaussian complex noise vector with zero mean and the identity covariance matrix I. Hence, the capacity of the secondary network is

$$
C_{s}=\log _{2}\left|\mathbf{I}+\mathbf{R}^{-1} \mathrm{HQH} \mathbf{H}^{*}\right|
$$

where $\mathbf{Q}=E\left(x_{s} x_{s}^{*}\right)$ transmit covariance matrix of $\mathrm{S}_{\mathrm{T}}, \mathbf{R}-$ noise plus interference covariance matrix of $S_{R}$ is:

$$
\mathbf{R}=\left[\mathbf{I}+\sum \mathbf{T}_{\mathbf{i}} \mathbf{Q}_{\mathrm{p}, \mathrm{i}} \mathbf{T}_{\mathbf{i}}^{*}\right]
$$

where $\mathbf{Q}_{p i}=E\left(\mathbf{x}_{p i} \mathbf{x}_{p i}^{*}\right)$-transmit covariance matrix of $\mathrm{P}_{\mathrm{T}}$.

\section{Advanced OSA-MIMO Model}

As it was stated before, the main problem of $\mathrm{CR}$ is determination of the channel state to avoid the interferences caused to $\mathrm{P}_{R}$ by $\mathrm{S}_{\mathrm{T}}$. The problem can be formulated as follows [10]

$$
\begin{aligned}
& \text { maximize } \quad \log _{2}\left|\mathbf{I}+\mathbf{R}^{-1} \mathbf{H Q H}^{*}\right| \\
& \text { subject to } \operatorname{Tr}(\mathbf{Q}) \leq \mathrm{P}_{\mathrm{T}} \\
& \text { and to } \operatorname{Tr}\left[\mathbf{G}_{\mathbf{k}} \mathbf{Q} \mathbf{G}_{\mathbf{k}}^{*}\right] \leq \Gamma_{\mathrm{k}} \quad \forall k
\end{aligned} \quad .
$$

where $\mathbf{G}_{\mathrm{k}}$ - channels from $\mathrm{S}_{\mathrm{T}}$ to $k$-th $\mathrm{P}_{\mathrm{T}} ; \Gamma_{\mathrm{k}}$ - interference power threshold at $k$-th $\mathrm{P}_{\mathrm{R}}$.

The results of simulation in Rayleigh fading are shown in Fig.3 [11]

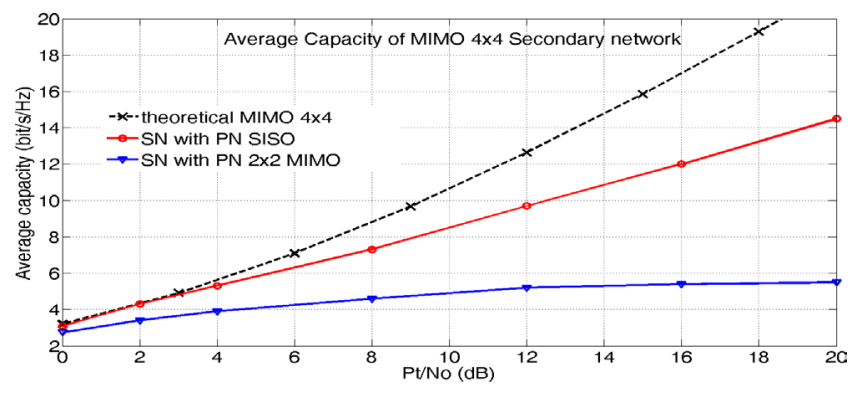

Figure 3. Capacity $\mathrm{C}_{\mathrm{S}}$ of the secondary network MIMO $4 \times 4$ under absence $(1)$ and presence $(2,3)$ primary networks SISO,MIMO

The dashed curve shows the theoretical unit capacity $\mathrm{C}_{\mathrm{S}} \mathrm{vs}$ SNR for an individual S network MIMO4x4. The next red curve shows the same capacity $\mathrm{C}_{\mathrm{S}}$ in presence of the primary network operating in SISO mode and the last curve (blue one) shows $\mathrm{C}_{\mathrm{S}}$ under both MIMO networks (primary $2 \times 2$ ). One can see that the most interesting is intermediate case. It shows that the secondary MIMO network can reach as much as $14 \mathrm{~b} / \mathrm{s} / \mathrm{Hz}$ supplementary capacity at $\mathrm{SNR}=20 \mathrm{~dB}$ under normal operation of primary SISO network in typical Rayleigh fading conditions

\section{Large Ad Hoc MIMO System}

Large Ad Hoc MIMO system composed of 100 transmit-receive nodes has been considered by Carvalho et al. [12]. In this experiment it is assumed that nodes are randomly displaced over a flat area of $1600 \times 1600 \mathrm{~m}$. The sensing range is $225 \mathrm{~m}$, the useful range $-150 \mathrm{~m}$. Each node can act both as transmitter or as a receiver. The two ray propagation model and the clear channel access mode (CCA) are assumed.

The goal of the approach taken by Carvalho et. al. is to assess the overall capacity of the system from the viewpoint of fading conditions and MIMO as well as from the effects of multiple access interference (MAI). The mathematical formulae are as follows:

$$
\left[\begin{array}{l}
y_{1} \\
y_{2}
\end{array}\right]=\left[\begin{array}{ll}
h_{11} & h_{12} \\
h_{22} & h_{22} \\
h_{11}^{*} & -h_{11}^{*} \\
h_{22}^{*} & -h_{21}^{*}
\end{array}\right]\left[\begin{array}{l}
s_{1} \\
s_{2}
\end{array}\right]+\left[\begin{array}{l}
n_{1} \\
n_{2} \\
n_{3}^{*} \\
n_{4}^{*}
\end{array}\right]=\mathbf{H}_{\mathrm{A}} \mathbf{s}+\mathbf{n}, \quad \mathbf{H}_{\mathrm{A}}^{\mathrm{H}} \mathrm{H}_{\mathrm{A}}=\|\mathbf{H}\|_{\mathrm{F}}^{2} \mathbf{I}_{2}
$$

where $\mathrm{y}_{1,2}$ - received signals at antennas 1,2 each for two sent signals $\mathrm{s}_{1}, \mathrm{~s}_{2}$ and noise; $\mathbf{I}_{2}$ - identity matrix $2 \times 2 ;\|\mathbf{H}\|_{\mathrm{F}}-$ 
Frobenius norm, $\|\mathbf{H}\|^{2}=\sum \mathrm{i} \sum \mathrm{j}|\mathrm{hij}|^{2}$.

By defining a new vector $\mathbf{z}=\mathrm{H}_{\mathrm{A}}^{\mathrm{H}} \mathbf{y}$ one gets

$$
\mathbf{z}=\|H\|_{F}^{2} \mathrm{I}_{\mathbf{2}} \mathbf{s}+\mathbf{n}^{\prime}, \quad \mathbf{z}_{\mathrm{i}}=\|H\|_{\mathrm{F}}^{2} \mathrm{~s}_{\mathrm{i}}+\mathbf{n}_{\mathbf{i}}^{\prime}
$$

where $\mathbf{n}^{\mathbf{\prime}}=\mathbf{H}^{\mathbf{H}}{ }_{\mathbf{A}} \mathbf{n}$ is a complex Gaussian noise vector.

The multiple access interference is added to the noise product $\mathrm{N}_{0}$ and the signal-to-interference-plus-noise ratio is as follows

$$
\operatorname{SINR}=\frac{\left\|\mathbf{H}_{\mathbf{i}}\right\|_{F}^{2} E_{s} / 2}{N_{\mathrm{O}}+\sum_{k=1}^{K}\left\|\mathbf{H}_{\mathbf{k j}}\right\|_{F}^{2} E_{s} / 2}
$$

where $i$-transmitted node; $j$ - receiving node; $\mathbf{H}_{\mathrm{kj}}$ - channel matrix from node $k$ to $j ; \mathrm{K}$ - number of simultaneous transmissions.

The simulation has been carried out on the basis of the popular $n s-3$ simulator [13]. The details of experiment assumptions are as follows:

- Energy threshold of reception $-73 \mathrm{dBm}$

- Clear Channel Access threshold -80.92 dBm

- Transmission power/noise figure $10 \mathrm{dBm} / 7 \mathrm{~dB}$

- Signal mode - DSSS, modulation - DPSK

- Transmission rate/packet size $1 \mathrm{Mbps} / 1412$ bytes

- One simulation run corresponds to $60 \mathrm{sec}$.

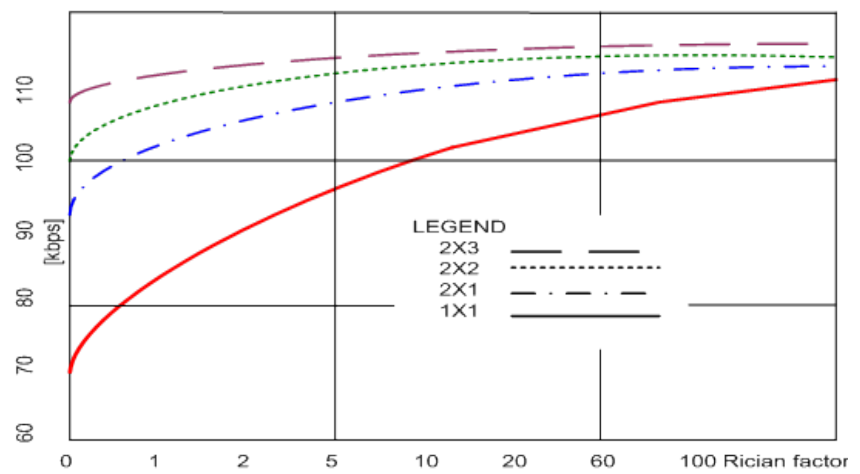

Figure 4. The growth of the Ad Hoc network's overall capacity vs. Rician factor (0 - pure Rayleigh) for different MIMO-s

The results of the experiment are shown in Fig.4. One can see that the overall growth of the system capacity due to the MIMO for Rayleigh fading is small and it reaches merely $\sim 70 \%$. If the Rice parameter of fading increases, this gain still decreases. It is also evident that using more than $2 \times 2$ antennas brings a negligible growth. According to the authors opinion the small gain obtained in the experiment is a result of completely random access to the common channel by all the users (MAI).

\section{Homogeneous MIMO Network}

Let us consider the network composed only of the MIMO channels and some number of special access points (OSA-routers). They contain all the necessary information on the current traffic and power distribution within the network. Similarly to the case presented in chapter 2 , the two groups of users are specified, the primary $\mathrm{P}$ and the secondary S. The only interference is the thermal noise and the Rayleigh fading. Hence, the supplementary capacity of the secondary network $S$ for equal access (50\%) is as follows:

$$
C_{s}=0.5 \log _{2}\left\{\operatorname{det}\left[\mathbf{I}_{\mathbf{M}}+(S N R / M) \mathbf{H H}^{\mathbf{H}}\right]\right\}
$$

Fig.5 shows the values of $\mathrm{C}_{\mathrm{S}}$ versus $\mathrm{SNR}$ obtained in the simulation. The results show that MIMO applied in both networks, $\mathrm{P}$ and $\mathrm{S}$, brings the small capacity $\mathrm{C}_{\mathrm{S}}$ in the secondary network. It approaches $5 \mathrm{~b} / \mathrm{s} / \mathrm{Hz}$ for MIMO3x3.

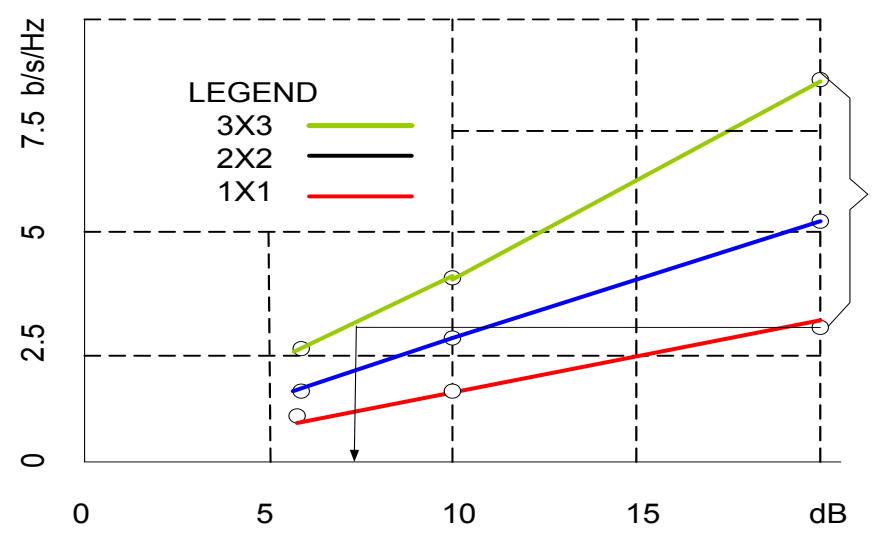

Figure 5. Supplementary capacity for two same rank OSA-MIMO networks operating under Rayleigh fading conditions

\section{Conclusion}

In the article a comparative analysis of primary-secondary OSA-MIMO systems operating in Rayleigh fading environment has been done from the viewpoint of the spectrum economy. The assumptions from this work are as follows:

1). The fluctuations of signals are due to attenuation and due to slow and flat Rayleigh fading.

2). The instant levels of signals are detected and spread over by the special OSA-routers.

3). The correlation between channels is neglected.

4). The information transmitted tolerate delays caused by channels fading and switching.

The obtained supplementary capacity $\mathrm{C}_{\mathrm{s}}$ in the secondary network depends on the organization of a system. The most interesting is the case when the secondary network is organized in MIMO mode, while the primary - in SISO mode. Then, the supplementary capacity $\mathrm{C}_{\mathrm{S}}$ reaches $8-14 \mathrm{~b} / \mathrm{s} / \mathrm{Hz}$ at $\mathrm{SNR}=20 \mathrm{~dB}$ for MIMO $2 \times 2$ and $4 \times 4$ antennas, resp.

It is worth to note that the capacity of an individual channel (without fading) provides merely $6,66 \mathrm{~b} / \mathrm{s} / \mathrm{Hz} / 20 \mathrm{~dB}$.

In all honesty, we have to admit that the above results are obtained in Rayleigh fading conditions. In the absence of fading $\mathrm{C}_{\mathrm{s}}=0$. However, such a condition never exists and the Rayleigh fading is only a model of the real world. As we 
already said, the licensed primary networks remain inactive over $90 \%$ of time (on average). This is much more than the Rayleigh model admits $(\sim 30 \%)$.

Starting from this point, the remaining problem of the spectrum economy shifts to the system of sensing and management instruments. It should answer the question when and where the free channels exist and how to use them. Therefore, the organization of the future wireless networks have to be modeled - to some extent - on the Internet philosophy [14].

\section{REFERENCES}

[1] J. Mitola and G.Q. Maguire, "Cognitive radio: Making software radio more personal", IEEE Pers. Commun. vol 4, no 4,1999

[2] B. Fette, Cognitive Radio Technology, Elsevier 2009

[3] S. Haykin, "Cognitive Radio: Brain-Empowered Wireless Communications," IEEE J. Sel. Areas Commun., vol. 23, no. 2, pp. 201-220, Feb. 2005.

[4] S. M. Alamouti, "A Simple Transmit Diversity Technique for Wireless Communications.," IEEE JSAC., vol. 16, no 8, 1998.
[5] S. Haykin, Adaptive Filter Theory, Prentice H. 1996

[6] R. Zhang, Y.-C. Liang, and S. Cui "Dynamic Resource Allocation in Cognitive Radio Networks: A convex optimization perspective," IEEE Signal Process. Mag., vol. 27, no. 3, May 2010.

[7] S. Astaneh and S. Gazor."Cooperative Spectrum Sensing...", IEEE Tran. Veh. Tech., no 3, June 2013

[8] R. Zhang, Y.-C. Liang, "Exploiting Multi-Antennas for Opportunistic Spectrum Sharnig in CR Networks," IEEE JSTSP., vol 2, no. 1, Feb. 2008

[9] J.Wang, G. Scutari, and D.P. Palomar, "Robust MIMO Cognitive Radio via Game Theory," IEEE Trans. Signal Process., vol. 59, no. 3, Mar. 2011.

[10] J. Mao et al.,"Power Allocation over Fading Cognitive MIMO Channels", IEEE TVT, no 3, 2012

[11] K. Kosmowski, J. Pawelec, "Performance Analysis of STC MIMO Systems over Correlated and Dynamic Channels", IEEE Com. Conf.,Poznan 2012

[12] M. Carvalho et.al., "An Approach for Discrete-Event Simulations of Alamouti System", J. Latex Class Files, no 1, 2007.

[13] www.nsnam.org.

[14] J. Pawelec et al.,"MIMO and CR Systems", Telecom. Review, no 1, 2013 (in Polish) 